\title{
Specific immunotherapy with mugwort pollen allergoid reduce bradykinin release into the nasal fluid
}

\author{
Radoslaw Gawlik¹, Alicja Grzanka², Barbara Jawor², Eugeniusz Czecior ${ }^{3}$ \\ ${ }^{1}$ Chair and Clinical Department of Internal Diseases, Allergology and Clinical Immunology, Medical University of Silesia, Katowice, \\ Poland \\ ${ }^{2}$ Clinical Department of Internal Diseases, Dermatology and Allergology in Zabrze, Medical University of Silesia in Katowice, Poland \\ ${ }^{3}$ Chair and Clinical Department of Otolaryngology in Zabrze, Medical University of Silesia in Katowice, Poland
}

Adv Dermatol Allergol 2016; XXXIII (4): 269-275

DOI: 10.5114/ada.2016.61602

\begin{abstract}
Introduction: A pathomechanism of allergic rhinitis is complex. A neurogenic mechanism seems to play a significant role in this phenomenon.

Aim: The evaluation of influence of specific immunotherapy of mugwort pollen allergic patients on the bradykinin concentration in the nasal lavage fluid.

Material and methods: The study included 22 seasonal allergic rhinitis patients. Thirty persons with monovalent allergy to mugwort pollen, confirmed with skin prick tests and allergen-specific immunoglobulin E, underwent a 3-year-long allergen immunotherapy with the mugwort extract (Allergovit, Allergopharma, Germany). The control group was composed of 9 persons with polyvalent sensitivity to pollen, who were treated with pharmacotherapy. Before the allergen-specific immunotherapy (AIT) and in subsequent years before the pollen seasons, a provocation allergen test with the mugwort extract was performed, together with collection of nasal fluids, where bradykinin concentration was determined according to Proud method.

Results: There were similar levels of bradykinin in both groups at baseline prior to therapy (AIT group: $584.0 \pm 87.2$ vs. controls $606.3 \pm 106.5 \mathrm{pg} / \mathrm{ml}$ ) and changes after allergen challenge $1112.4 \pm 334.8 \mathrm{vs} .1013 .3 \pm 305.9 \mathrm{pg} / \mathrm{ml}$ as well. The bradykinin concentration in nasal lavage fluid after mugwort challenge in 1 year was lower in the AIT group $(824.1 \pm 184.2 \mathrm{pg} / \mathrm{ml}$ vs. $1000.4 \pm 411.5 \mathrm{pg} / \mathrm{l} ; p<005)$ with a further significant decrease after the $2^{\text {nd }}$ and $3^{\text {rd }}$ year of specific immunotherapy. Significant reduction of symptoms and medications use was observed in hyposensitized patients.

Conclusions: A decreased level of bradykinin as a result of AIT suggests that some of the symptomatic benefits of AIT may be related to the reduced release of bradykinin into nasal secretions. These values correlate with clinical improvement within the course of treatment.
\end{abstract}

Key words: seasonal allergic rhinitis, allergen immunotherapy, mugwort, bradykinin, nasal lavage.

\section{Introduction}

Allergic rhinitis is the most often present allergic disease. Its incidence has doubled within the recent 10 years. It is currently estimated that about $14-18 \%$ of the population suffer from the allergic rhinitis and conjunctivitis, and its incidence proves an increasing tendency $[1,2]$. Allergens that trigger seasonal allergic rhinitis are pollens of grass, grains, trees and weeds. The only causative approach of treating allergic diseases with the potential to modify its course is allergen-specific immunotherapy (AIT) [1-3]. Despite numerous studies, a mechanism of this treatment method still remains not completely explained. The principle mechanism of AIT is induction of peripheral T cells tolerance and promotion of the formation of Treg cells. Exposure of sensitized patients to the allergen leads to cross-linking of a specific IgE antibody, bound to cell membranes of mastocytes, basophils, and to activation of inflammatory cells and a synthesis and the release of mediators, cytokines, and chemokines. This group of proinflammatory mediators responsible for

Address for correspondence: Ass. Prof. Radoslaw Gawlik MD, PhD, Chair and Clinical Department of Internal Diseases, Allergology and Clinical Immunology, Medical University of Silesia, 35 Ceglana St, 40-952 Katowice, Poland, phone: +48 601464786 , e-mail: radwags@poczta.onet.pl Received: 25.09.2015, accepted: 29.03.2016. 
the disease development also contains neuropeptides as of bradykinin, substance $P$, neurokinin $A$, kallikrein and others $[4,5]$.

A neurogenic mechanism plays a significant role in physiological regulation of nasal activity. Rich innervation of nasal mucous membrane together with plenty of immunocompetent cells, seem to be functionally interrelated. Furthermore, an immunoneurological interaction, as it has been proved by recent studies, plays a highly significant regulatory role in modulating an immunological response of allergic inflammation, and breaking of this balance contribute to development of allergic symptoms [4-9]. A certain role in development of inflammatory lesions in rhinitis pathophysiology is associated with bradykinin. This nanopeptide is released from $\alpha 2$ globulin (high- and low molecular kininogen - HMWK and LMWK) through enzymes, mainly serous and tissue kallikrein. Bradykinin works through stimulation of specific kinin B1 and $B 2$ receptors $[10,11]$. Immunohistochemical examinations proved presence of the $B 1$ and $B 2$ receptors on the epithelium, mucous glands, fibroblasts, vascular endothelium, vascular smooth muscles, venous sinusoids, macrophages $[8,12]$. Only the B2 receptors were confirmed to be present in peripheral nerves endings [8, 12].

Mediators secreted during an allergic inflammation could stimulate and modulate the activity of neurons present in the respiratory tract, and the other way round - the nervous system may exert certain influence on activity of the inflammation process through stimulating blood vessels vasodilation and transudation as well as inflammatory and immunological active cells $[4,7,13]$ Bradykinin, while stimulating the nerve endings, is responsible for some rhinitis characteristic symptoms. Nasal spraying of exogenous bradykinin causes sneezing, coughing, itching and produce watery secretion, blocking the nose in the majority rhinitis patients $[14,15]$. All these symptoms are significantly reduced as a result of allergen immunotherapy [1, 2]. Immunological modulation is distinctly responsible for that but the function of the basic nasal regulator - neurogenic is also impacted. Contribution of bradykinin in this process still remains unclear.

\section{Aim}

The aim of the present study was to assess the influence of allergen immunotherapy on bradykinin concentration in the nasal lavage fluid of mugwort allergic patients.

\section{Material and methods}

The study included 22 allergic rhinitis patients. Thirty persons were allergic to the mugwort pollen (8 women, 5 men, mean age: $35.30 \pm 8.2$ years old), treated in the Regional Allergy Clinic in Zabrze. Duration of the disease was $7.1 \pm 5.7$ years on average ( 3 to 17 years). The diagnosis was made on the basis of data from anamnesis, positive skin prick tests with inhalant allergens, and aslgE assay. During the season preceding application of the specific immunotherapy, there was unsatisfactory effectiveness of pharmacotherapy recorded in all patients (antihistaminic medications, nasal corticosteroids). The seasonal allergic rhinitis patients with confirmed monosensitization to mugwort were qualified to the AIT according to the EAACI recommendations [3]. None of those persons was desensitized previously. In the treatment standardized modified allergens - allergoids of mugwort, were used Allergovit (Allergopharma, Germany). The vaccination was applied in a perennial schedule for 3 years.

The control group consisted of 9 patients ( 5 women, 4 men, mean age: $32.2 \pm 6.9$ years) with seasonal allergic rhinitis, whose symptoms were controlled pharmacologically (nasal corticosteroids and antihistamines and oral antihistamines). They presented symptoms of the disease for $6.4 \pm 4.6$ years on average ( 4 to 16 years). The skin prick tests performed confirm a polyvalent allergy to grass, trees and mugwort pollens. The patients experienced clinical symptoms throughout the whole pollen season, increased within the mugwort pollen one. None of those patients was desensitized earlier.

Effectiveness of the treatment was evaluated on the basis of records entered into a diary all over the pollen season (from May to September). The following symptoms were evaluated: coughing, intensity of watery secretion, nasal blockage, lacrimation. Subjects recorded quantities of the medications, application of which was assessed according to the following criteria: antihistaminic medications, topical cromones -1 point each, nasal corticosteroids -2 points.

Evaluation of the course of the mugwort pollen season and pollen concentration in the Silesian region during the study was based on data obtained from the Allergen Research Center in Warsaw.

\section{Skin prick tests}

Skin prick tests with inhalant allergens were performed with allergen extracts of house dust mites (Dermatophagoides pteronyssinus and Dermatophagoides farinae), timothy grass, ryegrass, rye, alder, hazel, birch, plantain, mugwort, nettle, dog's and cat's dander, 0.1\% histamine, control solution (Allergopharma, Re, Germany).

Nasal lavage was performed according to the method described by Proud et al. [16] in all patients before desensitization, and after the first, second year and third year of allergen immunotherapy (ITA) always before the pollen season. Initial nasal lavage with physiological saline was made in order to remove cell elements and possible remaining mediators. Ten minutes later, $5 \mathrm{ml}$ of sterile saline solution pre-warmed to $37^{\circ} \mathrm{C}$ was instilled into both nostrils, then expelled into the collecting tube. Afterwards, the control solution (of the allergen solu- 
tions solvent) was sprayed into one nostril nasal channel, and $0.1 \mathrm{ml}$ of mugwort solution with a concentration of $5000 \mathrm{BE} / \mathrm{ml}$ into the opposite nostril (Allergopharma, Reinbek, Germany). After further $10 \mathrm{~min}$, the nose was again rinsed with physiological saline, recovered after $10 \mathrm{~s}$. The nasal lavage fluids were then transferred into conical tubes and, after volume measurement, stored on ice before further processing. Mean recovery of nasal lavage fluids was $2.6 \pm 0.5 \mathrm{ml}$. The obtained material was centrifuged at $4000 \mathrm{~g}$ for $15 \mathrm{~min}$ in the temperature of $4^{\circ} \mathrm{C}$, removing the cell elements. The supernatant was stored in the temperature of $-70^{\circ} \mathrm{C}$, until the assays were performed.

\section{Bradykinin assay}

Bradykinin was estimated in the recovered nasal lavage fluid using a method, described by Proud et al. [16]. In the radioimmunoassay Tyr8-bradykinin was used (Peninsula Laboratories, Belmont, CA) which was iodinated by the method of Hunter and Greenwood [16]. A $0.5 \mu \mathrm{g}$ aliquot of Tyr8-bradykinin was mixed with $0.05 \mathrm{M}$ phosphate buffer, $\mathrm{pH} 7.4$, and $5 \mu \mathrm{l}(0.5 \mathrm{mCi}) \mathrm{Na}{ }^{125}$ I (Amersham Corp., Arlington Heights, IL). The iodinated bradykinin was diluted 1 : 4 with 0.2 M Tris/0.01 M EDTA/0.1\% lysozyme, pH 6.4, and frozen in aliquots. Rabbit antiserum to bradykinin was applied. A $200 \mu$ volume of standard bradykinin or unknown, at suitable dilutions, was incubated with $100 \mu \mathrm{l}$ of labeled bradykinin (10,000 cpm) and $100 \mu$ l of antiserum ( $1: 140,000$ final dilution) for $20-24 \mathrm{~h}$ at $4^{\circ} \mathrm{C}$. Supernatants were removed and the radioactivity associated with the precipitate was counted. The assay was capable of detecting a minimum dose of 20 pg bradykinin/ml.

Total IgE concentration and mugwort specific lgE (allergen-specific immunoglobulin $E$ - aslgE) in the serum was estimated with use of a fluoro-immunoenzymatic FEIA CAP System (Phadia, currently Thermo Fisher Scientific).

\section{Statistical analysis}

Excel 5.1 spreadsheet was applied to collect the data. Statistica for Windows was used for statistical calculations. Group comparisons were performed by Wilcoxon analysis. The study of the correlations was analyzed by using the Spearman correlation coefficient.

\section{Results}

The majority of the treated patients $(8 / 13-61.5 \%)$ reported significant improvement and reduction of the symptoms after the course of allergen immunotherapy (Figure 1).

In the mugwort allergic patients, visual analog score (VAS) decreased already after the first year of treatment from 9.0 to 7.16 , i.e. by $20.4 \%$. In the control group, this

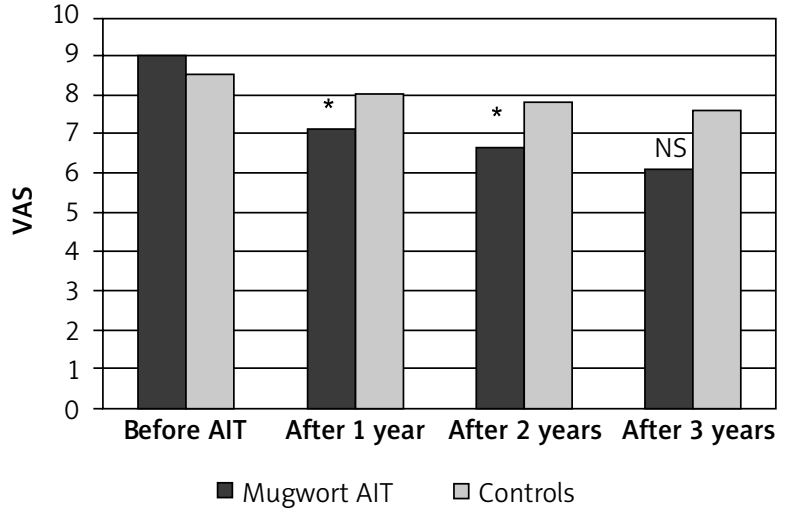

Figure 1. Mean values of symptoms score (VAS) in investigated patients during the 3 -year observation $\left({ }^{*} p<0.05\right)$

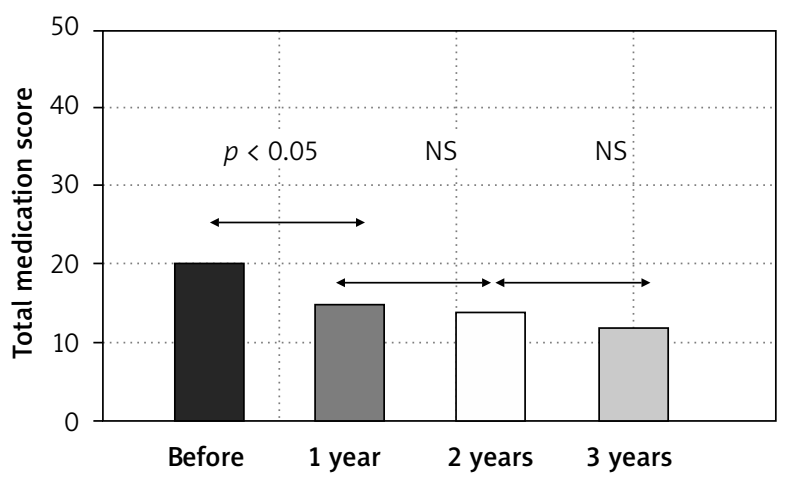

Figure 2. Total medication score in rhinitis patients allergic to mugwort $(n=13)$

parameter decreased after the first year only by $4.51 \%$, from 8.42 to 8.04 .

In a subjective evaluation of clinical improvement as a result of the 3-year course of immunotherapy, a 32\% decrease of VAS was recorded ( $\triangle \mathrm{VAS}-2.88)$. Within the control group, a minor improvement - by $10.2 \%$ - was observed ( $\triangle$ VAS - 0.90).

All of the desensitized patients lowered the number of previously used medications (Figure 2). Usage of medications dropped considerably after 1 year of SIT $(p<0.05)$, decreasing in subsequent years, however without statistical significance.

Mean values of tlgE and aslgE concentration in investigated groups of patients and subsequent years are presented in Table 1. None of them presented statistically significant changes.

\section{Bradykinin concentration in patients' nasal lavage fluid}

Bradykinin concentration in the nasal lavage fluid before the allergen challenge observed in subsequent years of the study was statistically insignificant (Table 2). 
Table 1. Concentration of total IgE and aslgE to mugwort in patients in subsequent years of observation

\begin{tabular}{lcccc}
\hline Group of patients & Before SIT [IU/ml] & After 1 year SIT [IU/ml] & After 2 years SIT [IU/ml] & After 3 years SIT [IU/ml] \\
\hline Mugwort tlgE & $192.4 \pm 35.2$ & $202.4 \pm 38.2$ & $198.2 \pm 28.6$ & $203.7 \pm 33.8$ \\
\hline Controls tlgE & $195.8 \pm 58.3$ & $192.8 \pm 61.0$ & $201.8 \pm 65.4$ & $199.4 \pm 61.6$ \\
\hline Mugwort - aslgE w6 & $19.6 \pm 7.8$ & $20.54 \pm 7.4$ & $19.72 \pm 7.1$ & $19.48 \pm 7.0$ \\
\hline
\end{tabular}

Table 2. Initial bradykinin concentration in the nasal lavage fluid of mugwort allergic rhinitis patients

\begin{tabular}{lcccc}
\hline Group of patients & Before SIT $[\mathrm{pg} / \mathrm{ml}]$ & After 1 year SIT $[\mathrm{pg} / \mathrm{ml}]$ & After 2 years SIT [pg/ml] & After 3 years SIT [pg/ml] \\
\hline Mugwort $(n=13)$ & $584.0 \pm 87.2$ & $524.8 \pm 46.2$, NS & $534.2 \pm 51.4$, NS & $474.2 \pm 63.5$, NS \\
\hline Controls $(n=9)$ & $606.3 \pm 106.5$ & $654.9 \pm 114.0$, NS & $626.4 \pm 82.8, \mathrm{NS}$ & $634.8 \pm 92.2$, NS \\
\hline
\end{tabular}

Table 3. Bradykinin concentration changes in patients' nasal lavage fluids after the mugwort allergen nasal provocation test $\left({ }^{*} p<0.05\right)$

\begin{tabular}{lcccc}
\hline Group of patients & Before SIT $[\mathrm{pg} / \mathrm{ml}]$ & After 1 year SIT $[\mathrm{pg} / \mathrm{ml}]$ & After 2 years SIT $[\mathrm{pg} / \mathrm{ml}]$ & After 3 years SIT [pg/ml] \\
\hline Mugwort $(n=13)$ & $1112.4 \pm 334.8$ & $824.1 \pm 184.2^{*}$ & $586.2 \pm 224.6^{*}$ & $564.4 \pm 208.4$ \\
\hline Controls $(n=9)$ & $1013.3 \pm 305.9$ & $1000.4 \pm 411.5$ & $960.0 \pm 287.9$ & $981.7 \pm 237.4$ \\
\hline
\end{tabular}

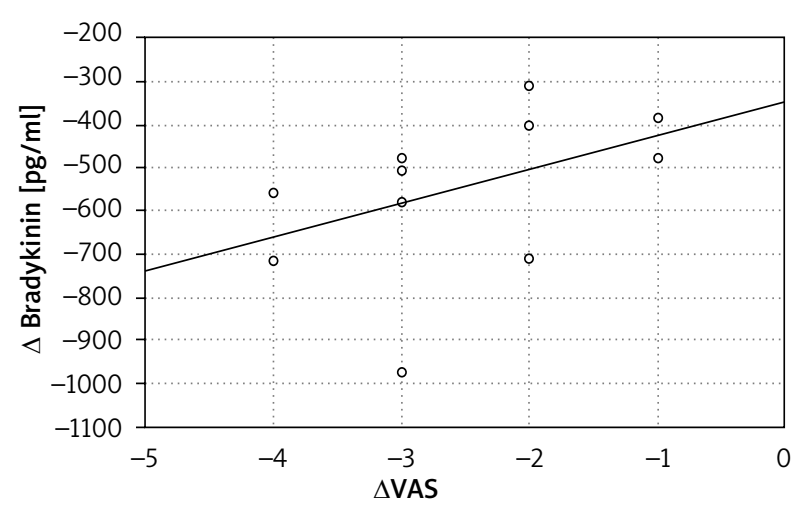

Figure 3. Correlation between increase of bradykinin nasal lavage fluid concentration after allergen provocation and symptoms severity after 3 year course of mugwort ITA $(r=0.49, p<0.05)$

Analysis of the basal bradykinin concentration in nasal lavage fluids before and after the 3-year treatment did not present any qualities of statistical significance $(p<0.062)$.

Statistically significant differences were observed while analyzing the increase of bradykinin concentration in nasal lavage fluid (NLF) after mugwort allergen challenge (Table 3).

Changes in the bradykinin concentration in the group of mugwort sensitized subjects were reduced af ter the first year of immunotherapy to $824.1 \pm 184.2 \mathrm{pg} / \mathrm{ml}$ (by $25.9 \%$ ); after the second year $586.2 \pm 224.6 \mathrm{pg} / \mathrm{ml}$ (by $47.3 \%$ ); after the third year $564.4 \pm 208.4 \mathrm{pg} / \mathrm{ml}$ $(-49.2 \%)$ and were statistically significant after the first and the second year of treatment $(p<0.05)$. Between the second and the third year of desensitization, there were no statistically significant changes observed $(p>0.05)$.

In the control group, mean changes in bradykinin concentration in NLF after allergen provocation in consecutive years was irrelevant: in the second year - 1000.4 $\pm 411.5 \mathrm{pg} / \mathrm{ml}$ (by $-1.27 \%$ ); in the third year $-960.0 \pm 287.9$ $\mathrm{pg} / \mathrm{ml}(-5.26 \%) ; \mathrm{IV}-981.7 \pm 237.4 \mathrm{pg} / \mathrm{ml}(-3.12 \%)$ (Table 3).

The severity of nasal symptoms and increase in the bradykinin concentration in nasal lavage fluids after NPT, presented a significant correlation already before the start of allergen immunotherapy $(r=0.70 ; p<0.05)$ and after the following years of treatment: after the first year of specific immunotherapy (SIT) $(r=0.66 ; p<0.05)$, after the second year of SIT $(r=0.47 ; p<0.05)$ and after the third year of SIT $(r=0.59 ; p<0.05)$.

A statistically significant correlation between changes of the bradykinin NLF concentration and symptoms score after 3 years of SIT $(r=0.49 ; p<0.05)$ was observed (Figure 3).

Severity of symptoms did not show any correlation with the serum mugwort specific IgE concentration and total IgE during the 3 -year observation $(p>0.05)$.

\section{Discussion}

Despite significant progress, which has taken place within recent years in the research exploring the allergic inflammation pathomechanism within the upper airways, there are still numerous elements that remain unclear. Results of the study showed that the neurogenic mechanism could have a role to play in this process. Neuropeptides released in the airways induce nasal allergic symptoms to a considerable extent, through stimulation 
of neuronal endings, inducing edema of the nasal mucosa and causing an increased permeability of vessels $[5,8,9,15,17]$. On the basis of available findings, it may be concluded that bradykinin is responsible for extending capillary vessels and its increased permeability, elevated generation of mucus, production of nitric oxide, and stimulation of nerve endings of non-myelinated type $C$ nerve fibers, and to release the neuropeptides, such as the $P$ and calcitonin gene-related peptide (CGRP) substance $[10,14,18,19]$. This efferent function of afferent type $C$ nerves is the axon response, and the effects of the released neurotransmitters are referred to as neurogenic inflammation.

Bradykinin presence in nerve endings, and its ability to stimulate mastocytes to release mediators, make this substance an important factor of the inflammatory process in airways [5, 12]. Our previous studies indicate a significant increase in the bradykinin concentration in the nasal secretion in the pollen season in allergic rhinitis patients $[16,20]$. Application of a selective antagonist of the bradykinin B2 receptor - icatibant - blocks these stimulations effectively [21]. Administration of icatibant in clinical trials allowed to decrease the nasal blockage, caused by allergen challenge in patients with allergic rhinitis [21]. Authors suggest the role for bradykinin in the development of allergic rhinitis.

In the majority of hyposensitized patients (61.5\%) a significant decrease in intensity of earlier recorded symptoms was observed. It was also expressed in the results of the nasal allergen provocation. Mugwort allergic patients already after the first year of specific immunotherapy presented, to a lesser extent, an increase of bradykinin concentration in NLF after allergen provocation. Dynamics of those changes correlated with clinical improvement expressed through a subjective evaluation of symptoms (VAS) $(r=0.47 ; p<0.05)$. The initial bradykinin concentration in nasal lavage fluids before the allergen challenge decreased over time in the SIT group, however these values were statistically nonsignificant and did not correlate with the clinical symptoms.

Analysis of concentration of allergen specific IgE to mugwort did not show any statistically significant changes. Furthermore, no changes of total IgE concentration were observed. These data are compliant with those obtained by Rogala et al. who did not record any significant changes in the concentration of allergen specific IgE [22] during the 3-year immunotherapy desensitization in house dust mite and grass pollen sensitive patients. These results differ from data presented by other authors that showed a drop in allergen specific IgE in the serum of patients, who completed a 3-5-year course of desensitization [23-25]. Studies of Rosewich et al. with immunotherapy against house dust mites showed significantly decreased slgE after subcutaneous immunotherapy (SCIT), but this reduction was not able to discriminate between responders and non-responders in the SCIT group [26].

It seems possible that the IgG immunoglobulins play a more significant role than it was previously thought. Interestingly Dokic et al. in their study demonstrated that IgG4 could inhibiting release of mediators [27]. IgG4 levels correlated with the TAME-enzyme activity, which is closely related with bradykinin formation $(r=0.85 ; p<$ 0.01). Different results were presented by Creticos et al. in their studies [28] who did not observe such a correlation in ambrosia allergic patients. In the group of patients with immunotherapy, the necessity to apply symptomatic treatment also decreased significantly already after the first year of the therapy. After the 3-year course of immunotherapy, only $25 \%$ of the desensitized patients required systematic pharmacological treatment during the pollen season. On the contrary, medication score in the control group did not change significantly. The most significant changes were observed in the first and second year of SIT, stabilizing in the subsequent year of desensitization. Blumberga et al. recently demonstrated that allergen-specific bronchial hyperreactivity (BHR) did not improve further in the second and third years of SCIT [29]. In the control group, there was no long-term effect of medications used, on the bradykinin concentration in the nasal lavage fluids, both in basal conditions and after the allergen challenge. Results of other studies show that use of topical corticosteroids decreases only a temporary release of mediators into nasal fluids. When treatment is discontinued, a concentration of mediators in nasal secretion returns rapidly to previous levels [30].

We did not observe any relation between the concentration of mugwort aslgE and the symptoms severity. These data are similar to the results of other authors, including Urbanek et al., Djurup et al. during desensitization of patients allergic to the Hymenoptera venom [31, 32], and Creticos et al. to ambrosia [33]. The immunomodulation mechanisms in allergic patients as a principle of allergen immunotherapy, have not been fully explained. Lack of correlation between the clinical improvement and changes in the slgE levels intrigue numerous researchers $[22,31,33]$. It is possible that apart from the mechanism that decrease the release of mediators by immunocompetent cells, there is another mechanism lowering tissue responsiveness. This mechanism may be managed by low molecular proteins and neuropeptides. There are observations suggesting that stimulation of the nervous system and development of neurogenic inflammation support development of hyperresponsiveness of the airways $[4,17]$. Bradykinin, released by numerous inflammatory cells, may be one of major contributors linking an allergic inflammatory reaction with neurogenic inflammation. The nervous and immunological systems cooperate within the process of the respiratory tract protection against pathogens. It was proved that a chronic 
inflammatory process in the airways led to an increased expression of bradykinin B2 receptors [10].

Previous results of our study showed an increased level of bradykinin concentration, within the pollen season, in the nasal secretion of allergic rhinitis patients in comparison with the group of healthy individuals [20]. An increase of vascular permeability in the respiratory tract of a rat, independent of mastocytes, suggests involvement of a non-immunological mechanism in etiology of vascular leakage [15]. Our study has demonstrated an impact of mugwort immunotherapy on the bradykinin concentration in NLF. No relationship between peptide concentrations and the aslgE levels implies involvement of other mechanisms that are not IgE related. Baumgarten et al. administering bradykinin to the nose, observed sneezing, rhinorrhea, nasal blockage and an increase in concentrations of P substance, in a dose-dependent manner [9]. It was a result of nerve endings stimulation, not of the influence on mastocytes. Studies by other authors also indicate that the mastocytes are not involved in the initial phase of neurogenic inflammation, which is characterized by an increased vascular permeability [34] but may be responsible for late secretion of bradykinin, substance $P$, and neurokinin A [35].

This study was not aimed to investigate the mechanisms underlying reduction of bradykinin release into NLF. The overall design of the study allows us only to speculate on the mechanism of modification of the bradykinin release within the course of allergen immunotherapy.

This study has several limitations. First, it was not a placebo-controlled study, what is a weakness in relation to assessment of clinical efficacy. In addition, a certain difficulty in interpretation of such kind of studies, is a small number of participants, limited by objective difficulties. Studies over neuropeptides in people, published in the literature, are based on small group of patients $[9,16,34]$, because of methodological difficulties (provocation tests, peptides markings in nasal secretion) and costs. We might speculate that bradykinin, along with other mediators is a factor linking immunological and nervous mechanisms in regulation of the nasal mucosa function. Bradykinin release, reduced as a result of allergen immunotherapy may contribute to diminution of the intensity of allergic inflammation.

We expect that future studies will answer the question about the role of neuropeptides in the immunological homeostasis of a human body, and their function in the mechanism of allergic reactions.

\section{Conclusions}

Allergen immunotherapy with mugwort allergoid of allergic rhinitis patients exerts suppressive influence on the neurogenic element of allergic inflammation, reducing secretion of bradykinin into nasal lavage fluids. Changes in the bradykinin concentrations in nasal lavage fluids do not correlate with the concentration of the allergen specific IgE.

\section{Conflict of interest}

The authors declare no conflict of interest.

\section{References}

1. Akdis M, Akdis CA. Mechanisms of allergen-specific immunotherapy. J Allergy Clin Immunol 2007; 119: 780-91.

2. Mailing HJ, Bousquet J. Subcutaneous immunotherapy for allergic rhinoconjunctivitis, allergic asthma, and prevention of allergic diseases. Clin Allergy Immunol 2008; 21: 343-5.

3. Zuberbier T, Bachert C, Bousquet PJ, et al. GA(2)LEN/EAACl pocket guide for allergen-specific immunotherapy for allergic rhinitis and asthma. Allergy 2010; 65: 1525-30.

4. Gawlik R. The role of neuropeptides in pathophysiology of rhinitis. Postep Dermatol Alergol 2010; 27: 162-5.

5. Mossiman BL, White M, Hohman R, et al. Substance P, CGRP, and vasoactive intestinal peptide increase in nasal secretions after allergen challenge in atopic patients. J Allergy Clin Immunol 1993; 92: 95-104.

6. Lundberg J, Hokfelt T, Martling T, et al. Substance P immunoreactive sensory nerves in the lower respiratory tract of various mammals including man. Cell Tissue Res 1984; 235 : 251-61.

7. Tonnesen P, Hindberg I, Schaffalitzky OB, et al. Effect of nasal challenge on serotonin, substance $P$ and vasoactive intestinal peptide in plasma and nasal secretion. Allergy 1988; 43: 310-7.

8. Said S. Proinflammatory and anti-inflammatory peptides. Marcel Dekker Inc., New York 1999.

9. Baumgarten CR, O'Connor A, Dokic D, et al. Substance P is generated in vivo following nasal challenge of allergic individuals with bradykinin. Clin Exp Allergy 1997; 27: 1322-7.

10. Baraniuk JN, Lundgren JD, Goff J, et al. Bradykinin receptor distribution in human nasal mucosa, and analysis of in vitro secretory responces in vitro and in vivo. Am Rev Respir Dis 1990; 141: 706-14.

11. Zhang Y, Adner M, Cardell LO. Up-regulation of bradykinin receptors in a murine in vitro model of chronic airway inflammation. Eur J Pharmacol 2004; 489: 117-26.

12. Shirasaki H, Kanaizumi E, Himi T. Immunohistochemical localization of the bradykinin B1 and B2 receptors in human nasal mucosa. Mediators Inflamm 2009, 102406, doi: 10.1155/2009/102406.

13. Proud D, Siekierski ES, Bailey GS. Human lung mast cells tryptase is a kinino-genase. Fed Proc 1987; 46: 932-9.

14. Austin CE, Foreman JC. A study of the action of bradykinin and bradykinin analogues in the human nasal airway. J Physiol 1994; 478: 351-6.

15. Kowalski ML, Didier A, Lundgren JD, et al. Role of sensory innervation and mast cells in neurogenic plasma protein exudation into the airway lumen. Respirology 1997; 2: 267-74.

16. Proud D, Togias AG, Naclerio RM, et al. Kinins are generated in vivo following nasal airway challenge of allergic individuals with allergen. J Clin Invest 1983; 72: 1678-84.

17. Kajekar R, Meyers AC. Allergic airway inflammation afferent neuron phenotype in guinea pig nodose ganglia. J Allergy Clin Immunol 1999; 101: s226.

18. Proud D, Reynolds CJ, LaCapra S, et al. Nasal provocation with bradykinin induces symptoms of rhinitis and a sore throat. Am Rev Respir Dis 1988; 137: 613-6. 
19. Thornton MA, Akasheh N, Walsh MT, et al. Eosinophil recruitment to nasal nerves after allergen challenge in allergic rhinitis. Clin Immunol 2013; 147: 50-7.

20. Gawlik R, Baumgarten C, Kunkel G, Pluskiewicz W. Kinin and TAME esterase in nasal lavage fliud of hay fever patients. Otolaryngol Pol 1994; 48: 116-20.

21. Austin CE, Foreman JC, Scadding JK. Reduction by Hoe 140, the B2 kinin receptor antagonist, of antigen-induced nasal blockage. Br J Pharmacol 1994; 111: 969-71.

22. Rogala B, Gluck J. Allergen-specific IgE in circulating immune complexes in patients with inhalant allergy undergoing specific immunotherapy. Invest Allergol Clin Immunol 1999; 9: 183-9.

23. Cantani A, Micera M. Significant decrease of IgE antibodies after a three-year controlled study of specific immunotherapy to pollen allergens in children with allergic asthma. Eur Rev Med Pharmacol Sci 2005; 9: 103-11.

24. Van Ree R, Van Leeuwen WA, Dieges PH, et al. Measurement of IgE antibodies against purified grass pollen allergens ( $\mathrm{Lol}$ p 1, 2, 3 and 5) during immunotherapy. Clin Exp Allergy 1997; 27: 68-74.

25. Bousquet J, Braquemond P, Feinberg J, et al. Specific IgE response before and after rush immunotherapy with a standardized allergen or allergoid in grass pollen allergy. Ann Allergy 1986; 56: 456-9.

26. Rosewich M, Arendt S, El Moussaoui S, et al. Bronchial allergen provocation: a useful method to assess the efficacy of specific immunotherapy in children. Pediatr Allergy Immunol 2013; 24: 434-40.

27. Dokic D, Nethe A, Kleine-Tebbe J, et al. Mediator release is altered in immunotherapy-treated patients: a 4-year study. Allergy 1996; 51: 796-803.

28. Creticos PS, Marsh DG, Proud D, et al. Responses to ragweed-pollen nasal challenge before and after immunotherapy. J Allergy Clin Immunol 1989; 84: 197-205.

29. Blumberga G, Groes L, Dahl R. SQ standardized house dust mite immunotherapy as an I immunomodulatory treatment in patients with asthma. Allergy 2011; 66: 178-85.

30. Schäper C, Gustavus B, Koch B, et al. Effect of fluticasone onneuropeptides in nasal lavage in persistent allergic rhinitis. I Investig Allergol Clin Immunol 2010; 20: 214-21.

31. Urbanek R, Kemeny DM, Richards D. Sub-class of IgE antibee venom antibody produced during bee venom immunotherapy and its relationship to long-term protection from bee stings and following termination of venom immunotherapy. Clin Allergy 1986; 15: 317-22.

32. Djurup DBK, Malling H, Sondrgaard I. The IgE and IgG subclass antibody response inpatients allergic to yellow jacket venom undergoing different regimens of venom immunotherapy. J Allergy Clin Immunol 1985; 76: 46-55.

33. Creticos PS, van Metre TE, Mardiney MR. Dose of response of IgE and IgG antibodies during ragweed immunotherapy. J Allergy Clin Immunol 1984; 73: 94-104.

34. Sertl K, Kowalski ML, Slater J, Kaliner MA. Passive sensitization and antigen challenge increases vascular permeability in rat airways. Am Rev Respir Dis 1988; 138: 1295-9.

35. Gawlik R, DuBuske L. Mediator release of neuropeptides after nasal provocation inperennial allergic rhinitis patients. Rhinology 2010; 48: 206-10. 